\title{
Worth the effort? Weighing up the benefit and burden of continuous positive airway pressure therapy for the treatment of obstructive sleep apnoea in chronic tetraplegia
}

\author{
Marnie Graco $\mathbb{D}^{1,2} \cdot$ Sally E. Green ${ }^{3} \cdot$ Julie Tolson $^{1} \cdot$ Bronwyn Stevens $^{1} \cdot$ Maree Barnes ${ }^{1,2} \cdot$ Alyssa Rigoni $^{1}$. \\ Sandra Henderson ${ }^{1} \cdot$ Carmel Nicholls $^{1} \cdot$ David J. Berlowitz $\mathbb{C}^{1,4}$
}

Received: 7 August 2018 / Revised: 22 September 2018 / Accepted: 4 October 2018 / Published online: 24 October 2018

(c) International Spinal Cord Society 2018

\begin{abstract}
Study design Mixed methods

Objectives Continuous positive airway pressure (CPAP) therapy is the recommended treatment for obstructive sleep apnoea (OSA). The aim of this study was to estimate CPAP adherence in people with tetraplegia and OSA, and to explore the barriers and facilitators to CPAP use.

Setting Hospital outpatient department in Melbourne, Australia

Methods People with chronic tetraplegia and OSA were commenced with auto-titrating CPAP and supported for 1 month. Semi-structured interviews were conducted with participants at one month and analysed thematically. CPAP usage was measured at 1, 6 and 12 months, with "adherent" defined as achieving more than $4 \mathrm{~h}$ average per night.

Results Sixteen participants completed the study ( $80 \%$ male; mean age $56(\mathrm{SD}=15)$ ). Mean nightly CPAP use at one month was $3.1 \mathrm{~h}(\mathrm{SD}=2.5 ; 38 \%$ adherent), and at 6 months and 12 months were $2.6 \mathrm{~h}(\mathrm{SD}=2.8 ; 25 \%$ adherent $)$ and $2.1 \mathrm{~h}$ $(\mathrm{SD}=3.2 ; 25 \%$ adherent). The perceived benefit/burden balance strongly influenced ongoing use. Burden attributed to CPAP use was common, and included mask discomfort, and physical and emotional problems. Adherent participants were motivated by the immediate daytime benefits to mood, alertness and sleepiness. There was a tendency to not recognise symptoms of OSA until after they were treated.

Conclusion CPAP use is challenging for people with tetraplegia, who experience substantial burden from using the device. When tolerated, the proximate benefits are substantial. People with tetraplegia need more intensive support for longer to help them overcome the burdens of CPAP and benefit from the treatment.
\end{abstract}

Electronic supplementary material The online version of this article (https://doi.org/10.1038/s41393-018-0210-z) contains supplementary material, which is available to authorized users.

Marnie Graco

marnie.graco@austin.org.au

1 Institute for Breathing and Sleep, Austin Health, Melbourne, Victoria, Australia

2 The University of Melbourne, Department of Medicine, Melbourne, Victoria, Australia

3 Monash University, School of Public Health and Preventive Medicine, Melbourne, Victoria, Australia

4 The University of Melbourne, Department of Physiotherapy, Melbourne, Victoria, Australia

\section{Introduction}

People with spinal cord injury (SCI) experience worse sleep than people without disability, as a consequence of a range of sleep disorders [1]. The most common sleep disorder in tetraplegia is obstructive sleep apnoea (OSA), with prevalence estimates from 56 to $97 \%$ [2-4]. OSA has been associated with worse quality of life and substantial neurocognitive deficits in people with tetraplegia $[4,5]$. Common symptoms of OSA in tetraplegia include snoring, daytime sleepiness and witnessed apnoeas [2]. It is thought that neuromuscular weakness, reduced lung volumes, disruptions to the autonomic nervous system, obesity, medications and supine sleeping position all contribute to higher OSA prevalence in tetraplegia [6].

Clinical practice guidelines for non-disabled populations and those with SCI recommend Continuous Positive 
Airway Pressure (CPAP) therapy for OSA [7, 8]. CPAP has been shown to effectively prevent the repetitive closure of the airway that occurs with OSA, and improve daytime sleepiness, quality of life and blood pressure in the nondisabled [9]. CPAP has also been found to improve daytime sleepiness in specialised populations such as stroke survivors and the elderly $[10,11]$.

Despite its benefits, CPAP effectiveness is limited by poor adherence to, and acceptance of, the therapy. Adherence to CPAP in the non-disabled is reported to range between 30 and $60 \%$ [12]. Two small studies have followed CPAP uptake in people with chronic SCI and OSA, reporting adherence rates to be $25 \%(2 / 8)$ and $67 \%(4 / 6)$ $[13,14]$. However in both studies adherence was determined through self-report, which has been found an unreliable method [12].

CPAP acceptance and adherence is a complex phenomenon that is not adequately explained through the quantitative analysis of risk factors. Several studies have attempted to understand the factors associated with CPAP adherence in the non-disabled using qualitative methodology [15]. Family and spousal support, beliefs about OSA, self-efficacy, and perceived barriers and facilitators to treatment are among the factors found to be influential $[15,16]$.

Factors associated with CPAP adherence in chronic tetraplegia have not been thoroughly investigated but are likely to be different to those of people without disability because of additional physical and psychosocial issues; including poor upper limb function, increased tactile sensitivity to the face, increased nasal congestion, reduced likelihood of bed partner, competing medical issues, sociodemographic differences, and additional causes of poor sleep such as pain and spasms. To our knowledge, there have been no qualitative studies investigating the unique influences on CPAP use in people with SCI, although the experience of sleep per se has been investigated using secondary analysis of qualitative data collected for a larger ethnographic study. This study identified poor sleep quality and quantity among participants, including frequent disturbances, and poor sleep patterns. Importantly, participants attributed their poor sleep to occupational disengagement, daytime fatigue and impaired cognitive functioning [17].

Given the high prevalence and impact of OSA in tetraplegia, a more in-depth understanding of the unique experiences of CPAP use in this population is required to develop targeted interventions that improve adherence. The aims of the study were: (1). To estimate adherence to CPAP in people with tetraplegia and explore associations between baseline factors and adherence. (2). To understand the individual experiences of using CPAP; including barriers and enablers to CPAP use.

\section{Methods}

\section{Design}

Mixed methods study, including an observational study and qualitative semi-structured interviews, with a cohort of people with tetraplegia and OSA commencing CPAP therapy. Refer to online supplement for additional methods.

\section{Setting}

Hospital outpatient department in Melbourne, Australia

\section{Participants and data collection}

Consecutive patients with chronic ( $>1$ year post-injury), traumatic tetraplegia (level T1 or higher; American Spinal Injuries Association (ASIA) Impairment Scale (AIS) A, B, C or D) attending spinal outpatient departments were recruited for a larger multicentre study, (Screening for OSA in Tetraplegia; SOSAT) [2]. SOSAT data utilised for this study included: demographic information, Karolinska Sleepiness Scale (KSS), Borg scale of nasal obstruction, Congestion quantifier five-item questionnaire, General self-efficacy scale (GSES) and sleep study data. (Online supplement). The sleep studies undertaken for the SOSAT study were unattended and performed in the participants' homes.

Upon receiving their sleep study results, all SOSAT participants recruited from the spinal outpatient clinic at the Austin Hospital in Melbourne, Australia were offered an outpatient appointment with a sleep physician. To access this service a referral from their treating doctor was required. Participants prescribed CPAP for OSA at this appointment were offered daytime auto-titrating CPAP implementation with an experienced sleep scientist at the CPAP clinic of the Austin Hospital, Melbourne. The autotitrating CPAP devices (AirSense 10 Autoset, ResMed, San Diego USA) wirelessly delivered real-time usage data to the treating clinical team. The sleep scientist contacted participants by telephone after three days, and at least weekly thereafter for four weeks. Additional support was available during the one-month period if required.

The sleep physician reviewed participants after four weeks. Immediately following this appointment, participants completed an in-depth semi-structured interview, the KSS, and a seven-item CPAP adverse events questionnaire [18]. The interview consisted of open-ended questions focusing on the experience of using CPAP, including barriers and enablers to CPAP use. CPAP usage data were obtained from the CPAP devices at one, six and 12 months. Interviews were conducted by an experienced interviewer with a clinical background in physiotherapy (MG). 


\section{Data analysis}

Quantitative data, including CPAP usage, pressure and leak, patient reported questionnaires and demographic information, were analysed and reported descriptively. CPAP usage data (mean hours per night) were reported over three time periods: Date of CPAP initiation to 1 month; $1-6$ months; and 6-12 months. CPAP "adherent" was defined as achieving more than four hours average per night over the entire period. Potential associations between baseline factors and mean nightly CPAP usage were explored with univariate linear regression analyses.

Interviews were audiotaped, anonymised and transcribed. Qualitative data were analysed using a general thematic approach [19]. Comparisons between "adherent" and "non-adherent" participants were made to identify patterns in the data and develop interpretations. (Refer to online supplement eTable1 for coding framework)

The study was approved by the Austin Health research ethics committee; AU/1/5FB02015. All applicable institutional and governmental regulations concerning the ethical use of human volunteers were followed during the course of this research.

\section{Results}

Of 44 eligible participants, 21 were referred to the sleep physician, 17 were prescribed CPAP, and 16 completed the study. (Fig. 1) Participants were predominantly male (81\%), mean age 56 years $(\mathrm{SD}=16)$ and mean body mass index (BMI) $27(\mathrm{SD}=6)$. On average they were two decades post injury, and had severe OSA (Table 1). Refer to online supplement for additional results.

On average, the CPAP implementation appointment took $1.7 \mathrm{~h}(\mathrm{SD}=0.4)$. Participants were telephoned an average of six times between CPAP implementation and the review appointment. Seven participants required additional appointments with the sleep scientist, including nine additional face-to-face appointments and three home visits. The average time the sleep scientist spent with each participant in the first month was $3.1 \mathrm{~h}(\mathrm{SD}=1.5)$, including implementation, telephone calls and review appointments. On average, participants tried three different models of CPAP mask. Most settled with nasal pillows $(n=10)$; while others opted for nasal masks $(n=3)$, and full face masks $(n=3)$; nine used a chinstrap.

At one month, mean nightly CPAP use was $3.1 \mathrm{~h}$, with $38 \%$ achieving at least $4 \mathrm{~h}$ per night. Mean nightly use dropped to $2.6 \mathrm{~h}$ at 6 months and $2.1 \mathrm{~h}$ at 12 months, with one quarter of the sample achieving at least $4 \mathrm{~h}$ per night in

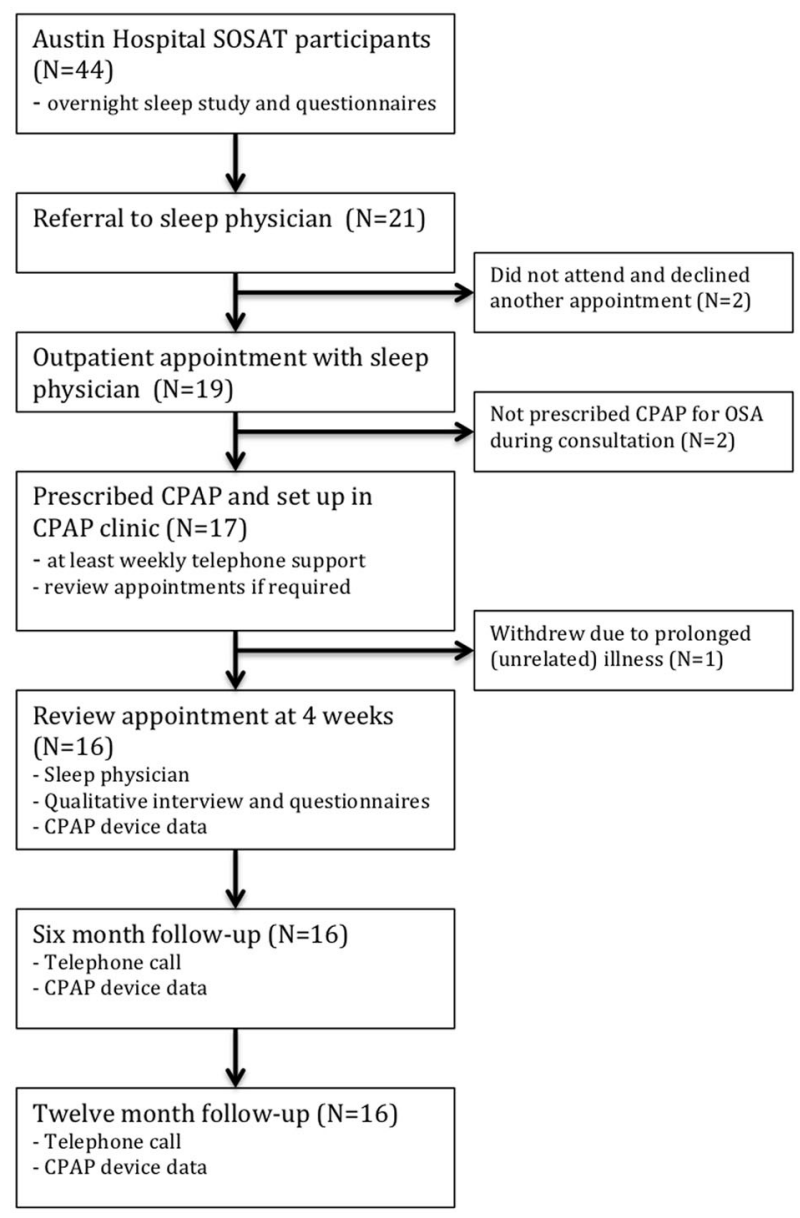

Fig. 1 Recruitment flowchart

these time periods. (Table 2) Individual participant CPAP usage data are displayed in Fig. 2 and eTable2 (onlinesupplement), showing six participants swapping their "adherent status" between months one and six (two became adherent and four became non-adherent). No participant changed adherent status after six months. By 12 months CPAP usage was distinctly bi-model and stable, with either high usage ( $>6 \mathrm{~h}$ per night) or low usage $(<3 \mathrm{~h}$ per night). Average $95^{\text {th }}$ percentile pressure ranged from 10 to $13 \mathrm{cmH}_{2} \mathrm{O}$ during the three time periods, while average $95^{\text {th }}$ percentile leak ranged from 21 to 26 litres/min. (Table 2)

CPAP use (average nightly hours) at six and 12 months were strongly associated with more hours spent with the sleep scientist in the first month and greater years since injury ( $p<0.05$; Online supplement eTable3,). Improvements in subjective daytime sleepiness in the first month appeared weakly associated with greater CPAP use at six $(p=0.06)$ and 12 months $(p=0.05$; eTable 3$)$. No associations between any other baseline variables with CPAP use were identified. 
Table 1 Demographic characteristics and other baseline data

\begin{tabular}{ll}
\hline & $N=16$ \\
\hline Age, years & $56.3(15.5)$ \\
Gender male, \%(n) & $81.3(13)$ \\
Time since injury, years & $21.0(14.9)$ \\
C1-C4, ASIA impairment scale (AIS) A,B,C, \%(n) & $6(1)$ \\
C5-C8, AIS A,B,C, \%(n) & $81(13)$ \\
T1-S3, AIS A,B,C, \%(n) & $0(0)$ \\
AIS D, at any level, \%(n) & $13(2)$ \\
C1-C4, \%(n) & $6(1)$ \\
C5-T1, \%(n) & $94(15)$ \\
AIS A, \% (n) & $25(4)$ \\
Body mass index (BMI), kg/m ${ }^{2}$ & $27.2(5.7)$ \\
Waist circumference, cm & $109.9(19.4)$ \\
Apnoea hyponoea index (AHI), events/hour & $49.5(30.0)$ \\
3\% Oxygen desaturation index (ODI), events/hour & $36.9(27.0)$ \\
Borg scale of nasal obstruction, score/10 & $1.0(1.4)$ \\
Congestion quantifier five-item questionnaire, score & $3.8(4.0)$ \\
range 0-20 & \\
General self-efficacy scale (GSES), score range 10-40 & $33.6(4.3)$ \\
Karolinska sleepiness scale (KSS), score range 0-9: & $4.3(2.1)$ \\
baseline & \\
Karolinska sleepiness scale (KSS), score range 0-9: & $2.9(2.1)$ \\
1 month review & \\
Number of CPAP adverse events: one month review & $3.4(1.8)$ \\
\hline Vales ar mos &
\end{tabular}

Values are mean (SD) unless otherwise indicated

\section{Qualitative interview results}

1. The burden of CPAP versus the benefit: "the lovehate relationship" (72 year old male, C6 AIS-D)

Most people in this study described the active, conscious decisions they made about whether the benefits of CPAP outweighed the burdens and hence whether to continue use. All participants experienced burdens and adverse events from using CPAP, and the trade-off between the perceived burden and the perceived benefit appeared to impact adherence to the therapy. For some, the perceived benefits and burdens were negligible, requiring the participant to decide whether the treatment was worthwhile. These participants spoke of their plans to discontinue CPAP for a specified period of time to better understand the benefits and to enable an informed decision of whether to continue. None of the participants in this group were CPAP users at 6 or 12 months.

"I think the only way to prove it to myself is if I go off the machine for two weeks and then go back on and just see the difference. And that will probably convince me one way or the other." (79 year old female, C5 AIS-C).

For others, both the immediate daytime benefits and

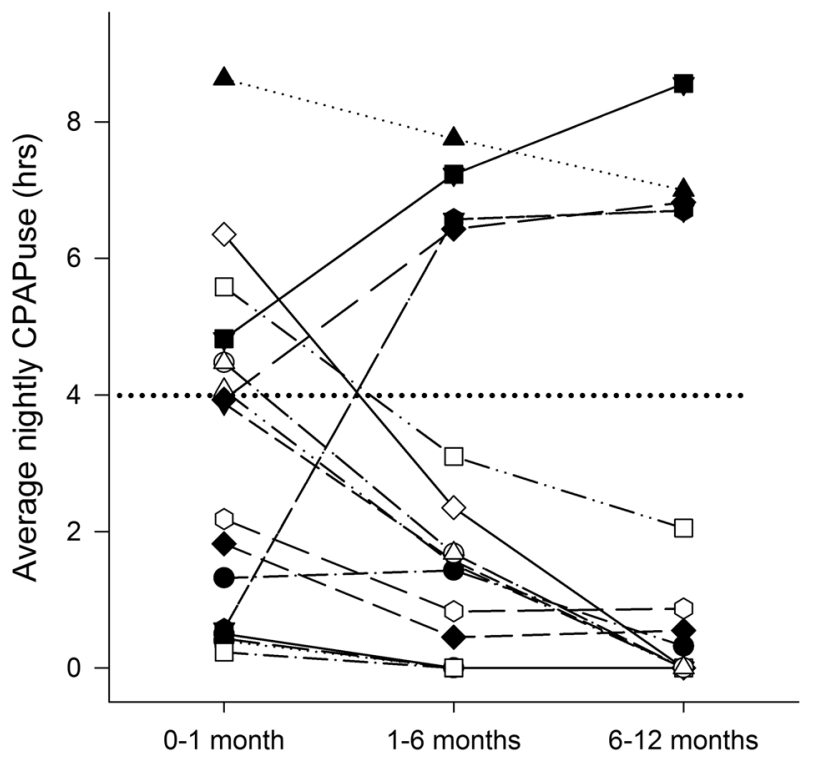

Fig. 2 Individual average nightly CPAP usage, measured at 1,6 and 12 months

the burdens were substantial. Three participants belonging to this group actively engaged with health professionals to troubleshoot issues and were able to overcome the burdens to become CPAP users. For those with high burden and little benefit, or low burden and substantial benefit, the decision of whether to continue with CPAP was easier, and their user status tended to be determined earlier. Unfortunately some participants did not experience any benefits from CPAP because they were unable to overcome the substantial burdens encountered from the beginning.

"I've never been able to fall asleep with it. I want to, but at the same time, am I fooling myself?" (49year-old male, C5 AIS-C).

The majority of participants were motivated by the immediate daytime benefits of having better sleep. Most were not concerned by the long-term health consequences of untreated OSA.

"For me [the main reason I am using CPAP is], so I'll have better days, physically and mentally." (65 year old female, C6 AIS-C)

"I know how the professionals would put it, and that's sleep apnoea can cause strokes or heart attack or whatever. I've never been one to worry about anything like that, and I'm probably still not. If I didn't use it, it wouldn't overly concern me in that respect." (72 year old male, C6 AIS-D).

This concept of burden versus benefit of CPAP is represented in Fig. 3, which describes four groups defined according to their perceived benefit and burden. Participants were retrospectively categorised to the most appropriate group (A-D) based on their 
Table 2 CPAP data (usage, $95^{\text {th }}$ percentile pressure and $95^{\text {th }}$ percentile leak)

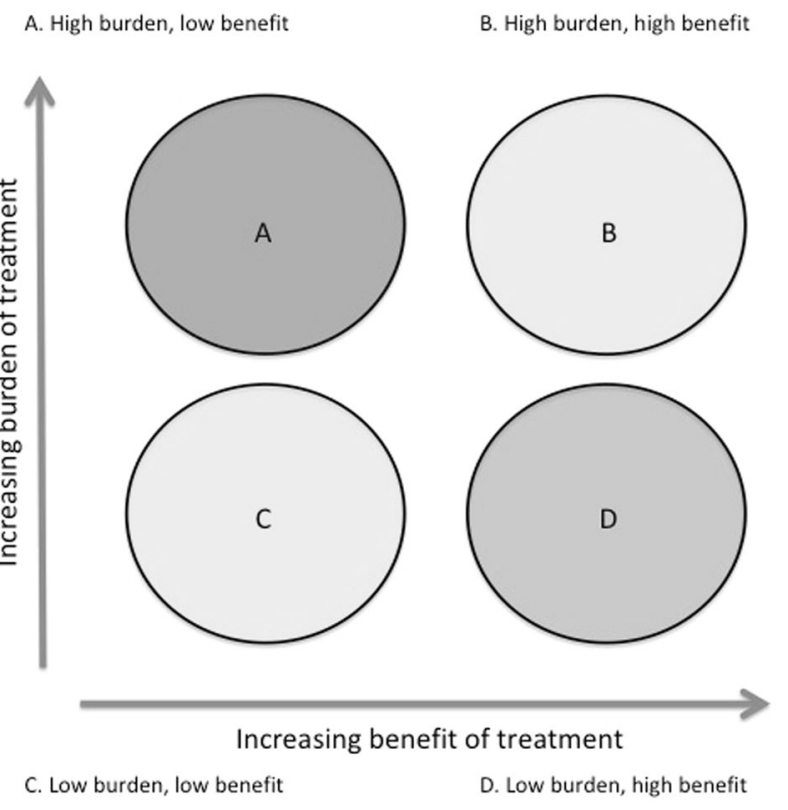

Fig. 3 CPAP burden versus benefit matrix

interview data, and average nightly CPAP use over the three time periods were calculated for each group (Online supplement eTable4). This pilot exercise demonstrated that those whose perceived benefit from CPAP was high (B\&D) had substantially higher CPAP use than those reporting low benefit (A\&C). Perceived burden impacted CPAP use to a lesser degree than perceived benefit.

The specific burdens and benefits are described in detail in the online supplement. Briefly, common burdens included issues with mask discomfort and fit, causing air leak, skin irritation and breakdown, and dry eyes and mouth. Considerable trial and error was required to overcome these issues. Participants also described psychosocial and emotional problems, including guilt about additional partner burden, claustrophobia, frustration and fear. Some participants had difficulty sleeping with the device. These problems were exacerbated by their physical disability, particularly limited upper limb function, and the already substantial workload associated with managing their SCI and the associated complications. At the 6 and 12 month review phone calls, several participants reported discontinuing CPAP when unwell in order to simplify their healthcare routines.

Benefits included improvements to sleep quality and sleep hygiene. Less daytime napping, reduced snoring, waking up refreshed, and fewer leg spasms at night were commonly reported. Other benefits included improvements in mood, energy levels, productivity, alertness and concentration.

Additional barriers and enablers to CPAP use, such as partner/family support and health professional support are also discussed in detail in the online supplement. Briefly, partner/family support appeared moderately important for some, but merely having this support did not ensure CPAP adherence. Overall, participants of this study were highly satisfied with the assistance they received from the health professionals involved in CPAP implementation.

\section{Barriers to OSA diagnosis}

While not an a priori focus of the interviews, two themes emerged relating to OSA diagnosis barriers.

a. Poor recognition of OSA symptoms prior to diagnosis and treatment

Several participants were surprised by the improvements they experienced from CPAP. Many felt they had incorrectly attributed daytime sleepiness to aging with a SCI, and did not realise the extent of their symptoms of OSA until after they had been treated. For these reasons many had not reported any symptoms of OSA to a health professional, and were initially surprised by their OSA diagnosis.

"No, I didn't realise, I just took it for granted that this is what happens as you get old with quadriplegia. I thought, 'well, just suck it up'. But now I realise what I've been missing out on." (71-year-old male, C6 AIS-A) 
"Personally, I was not aware of any problem whatsoever. I thought 'I'm not concentrating enough, I'm getting old, I haven't got enough to do.' That has turned around now, so it is obvious. It's made me aware of what the situation was." (72-year-old male, C6 AIS-D)

b. Overnight in-laboratory sleep studies are prohibitive to diagnosis

Most participants felt that having an overnight sleep study in a hospital or sleep laboratory would have been too difficult, and had it not been for the SOSAT study offering the test in their home, they would never have been diagnosed and subsequently treated for OSA. Their concerns centred on the potential disruption to their daily routines, and the inability of sleep laboratories to cater for the needs of people with disability.

"A couple of years ago the Austin contacted me and wanted me to come in to do it [overnight sleep study], but three kids, work, my wife's work; it was just too hard. I look back and now think I should have done it years ago." (43 year old male, C6 AIS-B)

"And being in a wheelchair, the thought of having to travel somewhere to go and do a sleep study and stay overnight, it's not very appealing. But obviously since you came out to the house it meant that I didn't have to leave home so it made it a lot easier." (49-year-old male, C5 AISB)

\section{Discussion}

CPAP adherence was low in this sample of people with chronic tetraplegia and OSA. Half of the participants were regularly using CPAP at 1 month, however this had reduced to a quarter by 6 months. Between 1 and 6 months, six $(38 \%)$ participants changed their "adherence status", from above to below $4 \mathrm{~h}$ per night or vice versa. Adherence status was then stable for all participants in the following 6 months. This suggests that CPAP patterns, which are established within a week in the non-disabled with OSA [20], can take up to 6 months in people with tetraplegia.

In this small study, we found that greater CPAP use at 6 months was strongly associated with more time spent with the sleep scientist in the first month. We speculate that participants who were more engaged were more likely to overcome the initial problems and continue with the treatment. Our qualitative data suggest it was the perceived benefit that drove the participants to seek solutions. These solutions required more time from the sleep scientist, but it was the perceived benefit, not simply "more therapy" that drove the improvements.
The perceived burdens relative to the perceived benefits of CPAP were identified as a major theme in this study. This same 'trade-off' has also been described in nondisabled populations [15]. However for people with tetraplegia, this decision is also made in the context of living with SCI. Most people with tetraplegia experience multiple secondary complications, with bladder and bowel dysfunction, spasms, pain, and pressure injuries being the most common [21]. The decision about whether CPAP is worthwhile was strongly influenced by the overall, ongoing burden of managing these SCI complications. Unfortunately the complexity of living with SCI often tipped the balance in the direction of discontinuing the therapy.

The concept of burden of treatment (BoT) has grown in the literature in the last decade in response to the rising prevalence of multi-morbidity. A recent meta-analysis of qualitative studies investigating BoT in people with chronic diseases found that the magnitude of the burden is related to the workload required, the individual's capacity, and the environmental context. Patients often prioritise treatments to reduce the workload, and try to integrate the treatments into their daily lives [22]. Data from our study are concordant with these findings. At the 6 and 12 month reviews, our participants commonly reported discontinuing CPAP during times of illness. This phenomenon has been reported previously. In another study investigating OSA treatment adherence in people with SCI, participants reported only tolerating bi-level positive airway pressure when their health was stable, and frequently suspended treatment during periods of illness [23].

Patient reported measures of BoT have been developed for people with multi-morbidity [24]. Rosbach et al [22] reason that the weight of BoT should be assessed in those with multi-morbidity, and health care providers should aim to reduce treatment burden where possible. Given the nature of SCI, BoT should arguably be considered in this population when prescribing new and challenging treatment regimes, such as CPAP.

The simple matrix assessing perceived burden and benefit from CPAP in the first few weeks of treatment (Fig. 3) could potentially be applied to predict long-term CPAP adherence. Further research could establish the clinical utility of this model to categorise patients into the four types of early CPAP users, in order to guide interventions to improve adherence. For example, those in the high burden, high benefit group (Group B) might be offered intensive support including home visits and/or additional appointments with health professionals to help them to overcome their substantial burdens. Given our finding that CPAP use takes longer to establish in this population, this support should be offered for a longer period of time. Potential strategies for those with low burden and low benefit (Group $\mathrm{C}$ ) might include more education about the longer-term consequences of OSA and benefits of CPAP 
treatment. Those with high burden and low benefit (Group A) could be offered alternative treatments for OSA; and finally, those with high benefit and low burden (Group D) could be reviewed periodically to monitor adherence and encourage continuation of the therapy during or after illness.

With the exception of reduced leg spasm, the daytime benefits described by participants of this study, such as improved sleep quality, reduced daytime sleepiness, and improvements in mood and cognition, were all common to other population groups [25]. In contrast, many of the burdens were either unique to people with SCI or augmented by their disability. Guilt about the additional burden using CPAP placed on partners was a major concern for many participants in this study. Conversely, qualitative research in adults without disability has identified that guilt about the impact on partners by not using the therapy is a motivator for use [26]. Fear of problems, particularly recurrent skin break down, was another significant barrier for participants in this study, potentially exacerbated from previous experiences of pressure injuries. Frustration was usually described in relation to the participant's inability to independently adjust the mask, and we hypothesise that claustrophobia is also magnified in this population because of reduced independence and hypersensitivity to the face. These additional and amplified burdens are likely to contribute to lower CPAP adherence in this population.

That participants in this study were not motivated by the long-term consequences of untreated OSA may reflect health information fatigue from managing multiple co-morbidities. In contrast, several qualitative studies in non-disabled populations have reported that fear of long-term consequences of OSA is an important motivator to CPAP use [16, 26].

Given the unique and complex burdens and motivations for CPAP use in tetraplegia, we speculate that noncomplicated OSA may be better managed within a specialist spinal unit. Specialised spinal units are generally responsible for the overall management of the person's SCI and associated complications, coordinating care with other specialists as required. Diagnosis and treatment of noncomplicated OSA is managed independently of respiratory/ sleep specialists in several spinal units around the world, although this is not the usual model. We hypothesise that patient outcomes and satisfaction would improve with either enhanced disability training for respiratory/sleep units, or enhanced OSA management training for spinal units. Either way, research into alternative models of OSA care for people with tetraplegia is needed.

Despite the low adherence to CPAP and the high treatment burden reported by many participants in this study, the daytime benefits for those adherent to the therapy appeared to be substantial. One quarter of our sample was adherent at 6 and 12 months, with average nightly use of approximately seven hours among these four individuals. Given the positive effect CPAP had on our adherent participants, our data would suggest that it remains a worthwhile therapy in this population with such high prevalence of OSA. Ultimately, a better therapy will replace CPAP as the first line treatment for OSA, but until then, research investigating interventions to reduce burden and improve adherence is warranted [7].

While not a focus of the study, barriers to OSA detection emerged as a major issue. Participants reported their reluctance to attend an overnight sleep study in a sleep laboratory, citing the potential disruption to healthcare routines and the inability of non-spinal health services to meet the needs of people with tetraplegia. Available data suggest that OSA is largely under-diagnosed and undertreated in SCI [6]. Our qualitative data also suggest that many patients are not being screened, diagnosed and treated for OSA, despite the high prevalence. A simplified ambulatory model for diagnosing moderate to severe OSA in tetraplegia has recently been published, offering an alternative to overnight sleep laboratory testing [2]. By overcoming this major barrier to OSA diagnosis, ambulatory diagnostic models have the potential to substantially increase diagnosis rates in this population.

\section{Limitations}

This was a small study of 16 people living with chronic tetraplegia. As such the quantitative analysis should be considered as exploratory and hypothesis generating. Only five of the 16 interviews were double-coded by two researchers (MG and AR), however the coding framework was revised after the first five interviews and guided the analysis of the remaining 11. Our sample of convenience did not allow for sampling until saturation of themes; nonetheless saturation was achieved with no new themes emerging for at least the last five interviews.

\section{Conclusion}

Adherence to CPAP is low among people with tetraplegia and OSA. However the benefits described in this study by the quarter that used it well were substantial. People with tetraplegia experience high burden from CPAP, exacerbated by their disability. More intensive support, for a longer period of time is recommended to help them to overcome these burdens and to allow them to be established on treatment. Individuals' adherence patterns were set by 6 months and remained relatively unchanged out to 12 months.

Acknowledgements The authors wish to thank ResMed for the donation of the AirSense 10 Autoset devices, and the people with 
spinal cord injury who generously offered their time to participate in this research.

Author contributions Study design: MG, SEG, JT, MB, DJB. Participant recruitment and data acquisition: MG, JT, BS, SH, CN, MB. Data analysis and interpretation: MG, SEG, AR, DJB. Manuscript preparation and approval of final version: all authors.

Funding MG was supported by an Australian Government National Health and Medical Research Council post-graduate scholarship (grant number 1114181) and an Australasian Spinal Cord Injury Network $\mathrm{PhD}$ scholarship.

Online supplement The online supplement contains the following information: additional methods (including participant recruitment and data collection; qualitative analysis and coding framework) and additional results (including individual participant CPAP data; univariate analysis of risk factors for CPAP use; barriers and enablers to CPAP use).

\section{Compliance with ethical standards}

Conflict of interest The authors declare that they have no conflict of interest.

\section{References}

1. Giannoccaro MP, Moghadam KK, Pizza F, Boriani S, Maraldi NM, Avoni P, et al. Sleep disorders in patients with spinal cord injury. Sleep Med Rev. 2013;17:399-409.

2. Graco M, Schembri R, Cross S, Thiyagarajan C, Shafazand S, Ayas NT, et al. Diagnostic accuracy of a two-stage model for detecting obstructive sleep apnoea in chronic tetraplegia. Thorax. 2018:thoraxjnl-2017-211131:1-8.

3. Leduc BE, Dagher JH, Mayer P, Bellemare F, Lepage Y. Estimated prevalence of obstructive sleep apnea-hypopnea syndrome after cervical cord injury. Arch Phys Med Rehabil. 2007;88:333-7.

4. Berlowitz DJ, Spong J, Gordon I, Howard ME, Brown DJ. Relationships between objective sleep indices and symptoms in a community sample of people with tetraplegia. Arch Phys Med Rehabil. 2012;93:1246-52.

5. Schembri R, Spong J, Graco M, Berlowitz D. Neuropsychological function in patients with acute tetraplegia and sleep disordered breathing. Sleep. 2017;40:1-6.

6. Sankari A, Martin JL, Badr MS. Sleep-disordered breathing and spinal cord injury: challenges and opportunities. Curr Sleep Med Rep. 2017;3:272-8.

7. Respiratory management following spinal cord injury: a clinical practice guideline for health-care professionals. Consortium for Spinal Cord Medicine; Paralyzed Veterans of America; 2005.

8. Qaseem A, Holty J-EC, Owens DK, Dallas P, Starkey M, Shekelle P. Management of obstructive sleep apnea in adults: a clinical practice guideline from the American College of Physicians. Ann Intern Med. 2013;159:471-83.

9. Giles TL, Lasserson TJ, Smith B, White J, Wright JJ, Cates CJ. Continuous positive airways pressure for obstructive sleep apnoea in adults. Cochrane Database of Systematic Reviews. 2006 .
10. McMillan A, Bratton DJ, Faria R, Laskawiec-Szkonter M, Griffin S, Davies RJ, et al. Continuous positive airway pressure in older people with obstructive sleep apnoea syndrome (PREDICT): a 12-month, multicentre, randomised trial. Lancet Respir Med. 2014;2:804-12.

11. Ryan CM, Bayley M, Green R, Murray BJ, Bradley TD. Influence of continuous positive airway pressure on outcomes of rehabilitation in stroke patients with obstructive sleep apnea. Stroke. 2011;42:1062-7.

12. Weaver TE, Sawyer AM. Adherence to continuous positive airway pressure treatment for obstructive sleep apnea: implications for future interventions. Indian J Med Res. 2010;131:245.

13. Burns SP, Little JW, Hussey JD, Lyman P, Lakshminarayanan S. Sleep apnea syndrome in chronic spinal cord injury: associated factors and treatment. Arch Phys Med Rehabil. 2000;81:1334-9.

14. Sankari A, Martin J, Bascom A, Mitchell M, Badr M. Identification and treatment of sleep-disordered breathing in chronic spinal cord injury. Spinal Cord. 2015;53:145-9.

15. Ward K, Hoare KJ, Gott M. What is known about the experiences of using CPAP for OSA from the users' perspective? A systematic integrative literature review. Sleep Med Rev. 2014;18:357-66.

16. Sawyer AM, Deatrick JA, Kuna ST, Weaver TE. Differences in perceptions of the diagnosis and treatment of obstructive sleep apnea and continuous positive airway pressure therapy among adherers and nonadherers. Qual Health Res. 2010;20:873-92.

17. Fogelberg DJ, Leland, Blanchard NEJ, Rich TJ, Clark FA. Qualitative experience of sleep in individuals with spinal cord injury. OTJR: Occup, Particip Health. 2017;37:89-97.

18. Chai-Coetzer CL, Luo Y-M, Antic NA, Zhang X-L, Chen B-Y, He $\mathrm{Q}-\mathrm{Y}$, et al. Predictors of long-term adherence to continuous positive airway pressure therapy in patients with obstructive sleep apnea and cardiovascular disease in the SAVE study. Sleep. 2013;36:1929.

19. Braun V, Clarke V, Terry G. Thematic analysis. APA Handb Res Methods Psychol. 2012;2:57-71.

20. Weaver TE, Kribbs NB, Pack AI, Kline LR, Chugh TK, Maislin $\mathrm{G}$, et al. 'Night - to-night variability in CPAP use over the first three months of treatment. Sleep. 1997;20:278-83.

21. Adriaansen JJ, Post MW, de Groot S, van Asbeck FW, StolwijkSwüste JM, Tepper M, et al. Secondary health conditions in persons with spinal cord injury: a longitudinal study from one to five years post-discharge. J Rehabil Med. 2013;45:1016-22.

22. Rosbach M, Andersen JS. Patient-experienced burden of treatment in patients with multimorbidity-a systematic review of qualitative data. PLoS ONE. 2017;12:e0179916.

23. Brown JP, Bauman KA, Kurili A, Rodriguez GM, Chiodo AE, Sitrin RG, et al. Positive airway pressure therapy for sleepdisordered breathing confers short-term benefits to patients with spinal cord injury despite widely ranging patterns of use. Spinal Cord. 2018;56:777-89.

24. Eton DT, Yost KJ, Lai J-s, Ridgeway JL, Egginton JS, Rosedahl $\mathrm{JK}$, et al. Development and validation of the Patient Experience with Treatment and Self-management (PETS): a patient-reported measure of treatment burden. Qual Life Res. 2017;26:489-503.

25. Luyster FS, Dunbar-Jacob J, Aloia MS, Martire LM, Buysse DJ, Strollo PJ. Patient and partner experiences with obstructive sleep apnea and CPAP treatment: a qualitative analysis. Behav Sleep Med. 2016;14:67-84.

26. Broström A, Nilsen $\mathrm{P}$, Johansson $\mathrm{P}$, Ulander M, Strömberg A, Svanborg E, et al. Putative facilitators and barriers for adherence to CPAP treatment in patients with obstructive sleep apnea syndrome: a qualitative content analysis. Sleep Med. 2010;11:126-30. 